\title{
Ants Can Expect the Size of the Next Element in a Geometric Sequence of Increasing or Decreasing Shapes, Only If This Sequence Is Present
}

\author{
Marie-Claire Cammaerts ${ }^{1} \&$ Roger Cammaerts ${ }^{2}$ \\ ${ }^{1}$ Independent researcher, retired from the Biology of Organisms Department, University of Brussels, Belgium. \\ ${ }^{2}$ Independent researcher, retired from the Natural and Agricultural Environmental Studies Department (DEMNA) of \\ the Walloon Region, Belgium.
}

Correspondence: Marie-Claire Cammaerts, independent researcher, 27, Square du Castel Fleuri, 1170 Bruxelles, Belgium.

Received: October 12, 2021

Accepted: November 10, 2021

Online Published: November 19, 2021

doi:10.5539/ijb.v13n2p37

URL: https://doi.org/10.5539/ijb.v13n2p37

\begin{abstract}
Having shown that the ant Myrmica sabuleti can expect the following number in an arithmetic sequence of increasing or decreasing numbers, we here investigated on their ability in expecting the size of the following element in an increasing or decreasing geometric sequence of shapes, otherwise identical. We found that the ants could anticipatively correctly increment or decrement a geometric sequence when tested in the presence of the learned sequence, but not without seeing the sequence in its learned sequential order. Such a behavior, i.e. perfectly choosing the next element of a sequence when in presence of that sequence but not otherwise, seems appropriate for the use of encountered cues while foraging and returning to the nest.
\end{abstract}

Keywords: anticipation, episodic memory, cognition, Myrmica sabuleti, operant conditioning, visual perception

\section{Introduction}

It has previously been shown that the workers of the ant Myrmica sabuleti Meinert, 1861 are affected by distance and size effects concerning number discrimination (Cammaerts \& Cammaerts, 2020). It was also shown that they present distance and size effects while discriminating elements of different physical sizes (Cammaerts \& Cammaerts, 2020a). Furthermore, it has been shown that they expect the following number in an increasing or a decreasing arithmetic sequence of numbers (Cammaerts \& Cammaerts, 2021a, b). We therefore wondered if these ants could foresee the next element in an increasing or a decreasing sequence of identical elements differing only by their size. We thus set up an experimental protocol and a design similar to that employed for revealing the ants' expectation of the following quantity in an arithmetic sequence of increasing or decreasing numbers, but allowing examining if they can anticipate the physical size of the next element in a geometric sequence. Before explaining our method and relating our results, we briefly report what is known about animals' expectative behavior and sequence learning.

Scrub jays (Aphelocoma californica) are able to foresee future needs of food independently of the current ones (Raby et al., 2007; Correia et al., 2007). Great apes (bonobos, chimpanzees, orangutans) save tools in order to use them in the future (Mulcahy \& Call, 2006), present self-control behavior for obtaining more in the future (Osvath \& Osvath, 2008) and may keep an object with the intention of exchange it later for food (Dufour et al., 2007; Osvath \& Persson, 2013; Bourjade et al. , 2014). Kea parrots (Nestor notabilis) are able to wait with the intention of receiving a more appreciated reward, presenting then 'forward-planning' and 'cost-effectiveness assessment' (Schwing et al., 2017). Squirrel monkeys (Saimiri sciureus), having no water at their disposal and having to choose between a lot and little food, chose the latter, avoiding by this behavior to suffer from thirst afterwards. Rats do not detain this ability (Naqshbandi \& Roberts, 2006). Concerning the invertebrates, bumblebee workers of Bombus appositus which collect nectar from flowers expect obtaining a sufficient amount of food in order to recover the energy consumed over their harvest. If this expected amount is not reached, the bumblebees stop collecting on these flowers and try to find other more furnished ones (Hodges, 1981). Honeybees trained to learn larger/smaller size rules could thereafter extrapolate such learned rules to novel stimulus sizes (Howard et al., 2017). As for the workers of the ant M. sabuleti, they are able to exhibit acquired conditioning in a later situation (Cammaerts \& Cammaerts, 2018). They can also foresee the time and location of the next food delivery (Cammaerts \& Cammaerts, 2016a, b). Only foragers aged 2 to 3 years and not young ants, detain expectative behavior (Cammaerts \& Cammaerts, 2015). As mentioned here above, they can expect if the next number of an increasing or 
decreasing arithmetic sequence will be larger or smaller and which exact number it will be (Cammaerts \& Cammaerts, 2021a, b).

Learning a sequence (not only of numbers but also of elements such as of landmarks, visual cues, sounds, etc.) is a capability observed and sometimes largely studied in several animal species. Let us quote the review of Kershenbaum et al. (2014) on acoustic communication as well as works on sequential learning of visual patterns by honeybees (Collett et al., 1993) and the ant Cataglyphis cursor (Chameron et al., 1998). The ant Gigantiops destructor was shown to be able to use sequential learning of the size of visual landmarks and generalize the learned rules by interpolating the relative size of novel encountered landmarks (Beugnon \& Macquart, 2016). Concerning M. sabuleti workers, let us recall that they can learn an arithmetic sequence (Cammaerts \& Cammaerts, 2021a, b).

Our present aim is to know if M. sabuleti workers could expect (foresee) the size of the next element in an increasing or decreasing geometric sequence of previously perceived elements. The experimental design and protocol being partially similar to previous ones (Cammaerts \& Cammaerts, 2021a, b), they are here briefly described.

\section{Material and Methods}

\subsection{Collection and Maintenance of Ants}

The experiments were conducted on two colonies of $M$. sabuleti collected at Marchin (Condroz, Belgium) in an abandoned quarry. Each colony was kept in one to three glass tubes, the proximal part of each tube serving as a habitat, separated by a cotton plug from a distal section serving as a water reservoir. The tubes of each colony were deposited in a tray $(30 \mathrm{~cm} \times 15 \mathrm{~cm} \times 5 \mathrm{~cm})$, a thin layer of talc covering its borders in order to avoid the escaping of ants. These trays were the ants' foraging areas, in which they were trained and received their food. Food was delivered as pieces of Tenebrio molitor larvae (Linnaeus, 1758) given three times per week as well as sugar water continuously delivered in small tubes plugged with cotton. The ambient temperature equaled ca $20^{\circ} \mathrm{C}$, the humidity ca $80 \%$, the luminosity ca 330 lux while working on ants, and the electromagnetism ca $2 \mu \mathrm{Wm}^{2}$, these values being suitable to the species. The ants are here often named workers or nestmates as commonly do researchers on social insects.

\subsection{Experimental Planning}

Two experiments were successively made with a time interval of four days between them. The first experiment (Experiment I) aimed to examine the ants' potential ability in expecting the size of the following element of an increasing geometric sequence, while the second experiment (Experiment II) aimed to examine if ants could expect the size of the next element of a decreasing geometric sequence. Each of these two experiments was made of two parts: part 1 was devoted to the training of ants to the geometric sequence; part 2 consisted in checking if the ants could expect the element having the correct following size. As the M. sabuleti workers are able to distinguish all the colors (Cammaerts, 2007), the elements used during Experiment I were blue squares; those used during Experiment II were yellow squares. Readers are advised to look at Figures 1 and 2 for an easier understanding of the experimental method.

\subsection{Cues Presented to the Ants}

The cues (elements) used during the investigation about an increasing geometric sequence (Experiment I) were blue squares with an area equaling 1,2, 4 and $8 \mathrm{~mm}^{2}$ for the first part of the experiment and, moreover, 16 and $32 \mathrm{~mm}^{2}$ for the second part of the experiment. The cues presented during the investigation about a decreasing geometric sequence (Experiment II) were yellow squares with an area equaling 32, 16, 8 and $4 \mathrm{~mm}^{2}$ for the first part of the experiment and, moreover, 2 and $1 \mathrm{~mm}^{2}$ for the second part of the experiment. The area of the presented squares thus geometrically increased or decreased according to an increment of 2 , and their side as well as their perimeter geometrically increased or decreased according to an increment of $2^{1 / 2}$.

Each cue was drawn inside of a square $(2 \mathrm{~cm}$ x $2 \mathrm{~cm})$ using Microsoft Word® software. All of them were printed on white paper, and tied to the front face of a stand using extra transparent sticky paper. This was done four days before starting the experiments to avoid the possible remaining of odor. Each stand was made of strong white paper $\left(250 \mathrm{~g} / \mathrm{m}^{2}, \mathrm{Steinbach} \otimes\right.$, Malmedy, Belgium). They had a vertical part $(2 \mathrm{~cm} \mathrm{x} 2 \mathrm{~cm})$ and were kept vertically thanks to a dully folded horizontal part $[2 \times(1 \mathrm{~cm} \times 0.5 \mathrm{~cm})]$ (this can be seen in Figures 3,4). Stands with no cue tied on them served as cues to avoid during training; they were located far from any reward while the stands with the cue to memorize were located aside the nest entrance. The stands and their cue used for the tests performed during the first part of the experiments and during the second part of the experiments were novel, but identical to those used for training. 


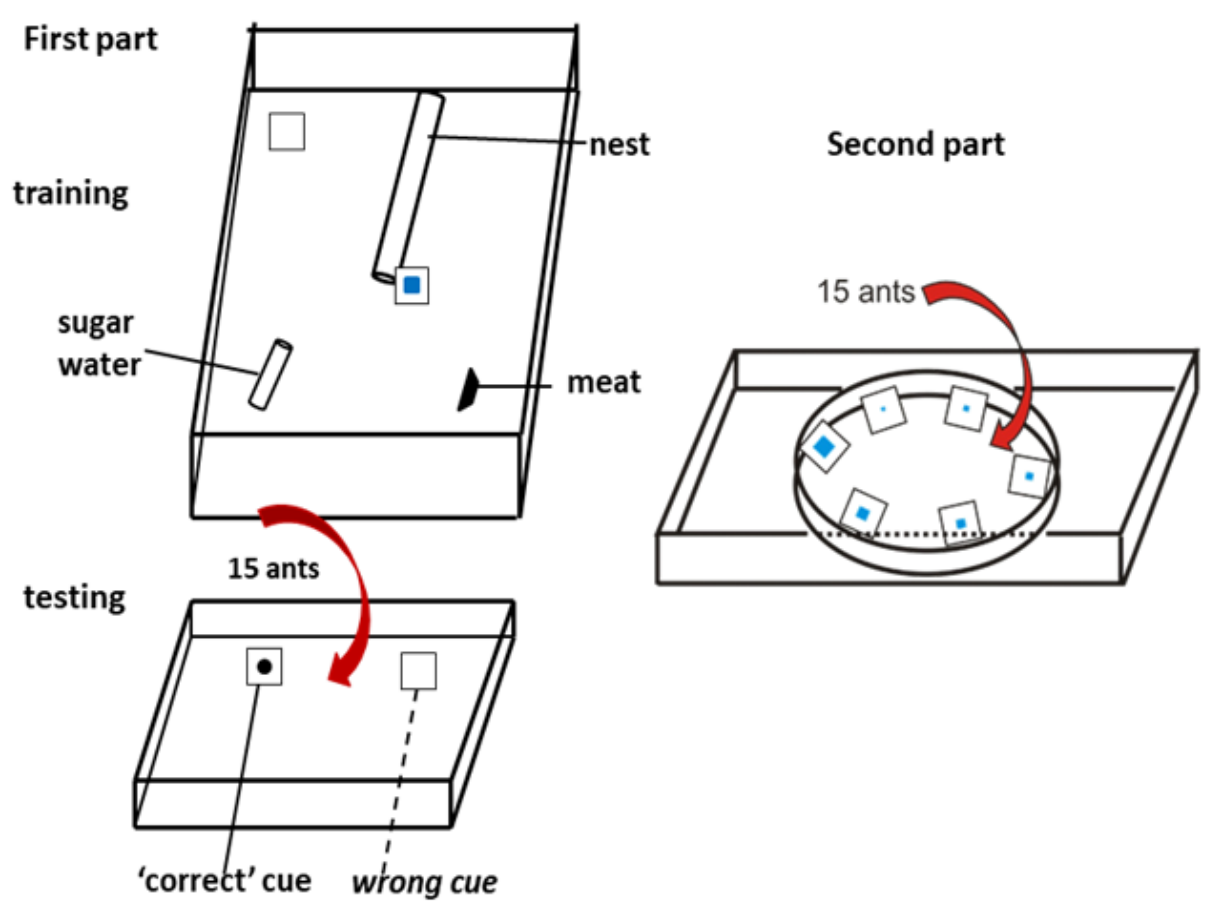

Figure 1. Experimental design used for performing the first and second parts of the experiments

Only one experiment made on one colony is here schematized but two experiments (Experiment I, with an increasing sequence using blue squares and Experiment II, with a decreasing sequence using yellow squares) have been performed, each of them conducted on two colonies. The second part of each experiment was made twice, firstly for Experiment I and secondly for Experiment II with the cues in their sequential order, secondly for Experiment I and firstly for Experiment II with the cues in a random order. A summary of the planning is given in Figure 2, and photos of the experiments are shown in Figures 3 and 4.

Experiment I: increasing geometric sequence, blue squares

First part: at days $1,3,5,7$, successive trainings towards squares of $1,2,4,8 \mathrm{~mm}^{2}$ at days $2,4,6,8$, successive tests in front of squares of $1,2,4,8 \mathrm{~mm}^{2}$

Second part: at day 9 ,

1. testing simultaneously in front of squares of $1,2,4,8,16,32 \mathrm{~mm}^{2}$ circularly presented in this sequential order 2. testing simultaneously in front of squares of $2,1,16,4,32,8 \mathrm{~mm}^{2}$ circularly presented in this random order

Experiment II: decreasing geometric sequence, yellow squares

First part: at days $1,3,5,7$, successive trainings towards squares of $32,16,4,2 \mathrm{~mm}^{2}$

at days $2,4,6,8$, successive tests in front of squares of $32,16,4,2 \mathrm{~mm}^{2}$

Second part: at day 9 ,

1. testing simultaneously in front of squares of $1,16,4,32,2,8 \mathrm{~mm}^{2}$ circularly presented in this random order

2. testing simultaneously in front of squares of $32,16,8,4,2,1 \mathrm{~mm}^{2}$ circularly presented in this sequential order

Figure 2. Experimental protocol

Details are given in the text, a partial schematic representation of the experimental design is provided in Figure 1, and photos of the experiments can be seen in Figures 3 and 4. 


\subsection{Experimental Design and Protocol}

\subsubsection{First part of the Experiments I and II}

At days 1, 3, 5, and 7 respectively, the first, the second, the third then the fourth element of the increasing or decreasing sequence was set aside the nest entrance and a blank stand was set away from any reward, the ants undergoing operant conditioning and having the possibility to memorize the four successively presented cues and their occurrence over time. Over these successive trainings to a sequence of squares of different dimensions, the ants of each two colonies sighted near the delivered cues were counted six times over each two days of each cue presentation (number of counts for each cue $=6 \times 2 \times 2$ ) and the mean of these 24 counts was established. These countings allowed to check if the ants equally and sufficiently saw the different presented cues. The four means obtained for the different cues were compared to the numbers that would result from an equal foraging around the four successively presented cues by using the $\chi^{2}$ non-parametric goodness-of-fit test. The means of these counts are given in Tables 1 and 2.

For assessing the ants' conditioning to each presented cue, the ants were tested on days 2, 4, 6, 8 in an own tray devoted to testing inside of which the 'correct' and the 'wrong' cues were randomly set one on the left and the other on the right. For conducting a test on a colony, 15 ants were deposited inside their tray devoted to testing. The ants freely moved in the tray and approached each two cues, staying 2 to 20 seconds near those of their choice. The ants present at a distance less than $2 \mathrm{~cm}$ from each cue were punctually counted 20 times (every 30 seconds) over 10 experimental minutes. On the basis of the 40 counts ( $20 \times 2$ colonies) obtained for each cue, the proportion of ants choosing the 'correct' cue (the one set aside the nest entrance during training), i.e. the ants' conditioning score, was calculated. As soon as a test was ended, the 15 ants were set back in their foraging area near their nest. For each cue, the 20 numbers obtained over time for each of the two colonies were added together in a corresponding manner and then chronologically added by four. This provided 5 successive groups of numbers. These five groups corresponding to the 'correct cue' were compared to the five groups corresponding to the 'wrong' cue (the one set away from any reward during training) by using the non-parametric matched-pairs signed-ranks test of Wilcoxon. The one-tailed $p$ value was read in the table for small sample sizes in Siegel and Castellan (1988). The non-parametric $\chi^{2}$ test was used to compare the distributions of the numbers of ants sighted near the 'correct' and the 'wrong' cues. The results of these two statistical analyses are given in the 'Testing' part of Tables 1 and 2. The obtained $p$ values did not change if the Benjamini-Hochberg adjusting procedure for multiple comparisons with a false discovery rate of 0.05 was used (McDonald, 2014).

\subsubsection{Second Part of Experiments I and II}

For making the second part of the experiments I and II, on day 9, the ants were tested in a circular enclosure made of Steinbach ${ }^{\circledR}$ paper, of $3 \mathrm{~cm}$ height, $15 \mathrm{~cm}$ in diameter, and $47.1 \mathrm{~cm}$ in perimeter and set in a tray $(23 \mathrm{~cm} \mathrm{x} 15 \mathrm{~cm} \times 5 \mathrm{~cm})$. For building each enclosure, $2.2 \mathrm{~cm}$ was added to the required perimeter for seam allowance. Six cues were deposited all along the rim of these enclosures: the four squares used during training, the correct following square of the increasing or decreasing sequence, and a 'wrong' square, the one that followed the correct one. More precisely, for the increasing sequence, the ants were tested in front of blue squares of $1,2,4,8,16$ and $32 \mathrm{~mm}^{2}$, and for the decreasing sequence, the ants were tested faced to yellow squares of 32, 16, 8, 4, 2 and $1 \mathrm{~mm}^{2}$. The cues used to test the ants were not the ones used during training, but newly made cues. In the first presentation for Experiment I and in the second for Experiment II, the cues were located as written here above, i.e. in the order they had been sighted over the training so that the ants could perceive the sequence to which they had been trained. The ants were thus confronted at the same time to the sequential cues learned during training, to the correct following one, and to its next one, all these cues being circularly located and presented in their sequential order. In the second presentation for Experiment I and in the first for Experiment II, a similar experiment was made with the cues no longer presented to the ants in their sequential order, but in a random order to check if ants trained to a geometric sequence could increment the last sighted element in the absence of the learned sequential order of the elements. To locate the cues in a random order, we used the order given by the last non-redundant digit of successive phone numbers written in a phone book. A time period of 20 minutes elapsed between tests 1 and 2.

To conduct a test on a colony, 15 ants were transferred in the center of the enclosure and the ants sighted near each of the six cues were counted 20 times over 10 minutes. The numbers obtained for each cue and each two colonies were added (see Tables 1 and 2, lower part). These six numbers, as well as the numbers corresponding to the two cues not presented in the first part of the experiment, but only in this second part, were compared to the numbers that would result from an equal visit to the six cues by using the non-parametric $\chi^{2}$ goodness-of-fit test (Siegel \& Castellan, 1988). The two numbers that were presented for the first time during this second part of the experiment were compared in the same manner. If the ants essentially visited the correct following cue of the increasing or decreasing sequence of squares, then they correctly expected the following dimension of the geometric sequence; if statistically they did not more visit the correct following cue than the other cues, then they did not duly increment the dimension of the next element of the sequence. 


\section{Results}

\subsection{Ants' Potential Ability of Incrementing an Increasing Geometric Sequence}

Numerical and statistical results are given in Table 1, and photos are shown in Figure 3.

During the first part of the experiment, the trained ants were sufficiently numerous near the cues they were expected to learn for perceiving and memorizing them. Also, they were statistically equally numerous near the four successively presented cues (difference with the mean value of the four obtained numbers: $p>0.995)$. Successively tested in front of each of the four presented squares versus zero square, the ants essentially reacted to the squares (Wilcoxon tests: $p=0.031$; $\chi^{2}$ tests: $p<0.001$ ). These results are graphically presented in Figure 5, upper left part. The ants' response to the first presented square was of high level $(91.43 \%)$. That to the second presented square was the lowest $(74.04 \%)$, and those to the third and the fourth presented squares were of intermediate level $(83.67 \%$ and $84.76 \%)$. These different levels of conditioning are explained in the Discussion section.

Table 1. Ants' learning then incrementing an increasing geometric sequence of blue squares

First part: learning the sequence

Training

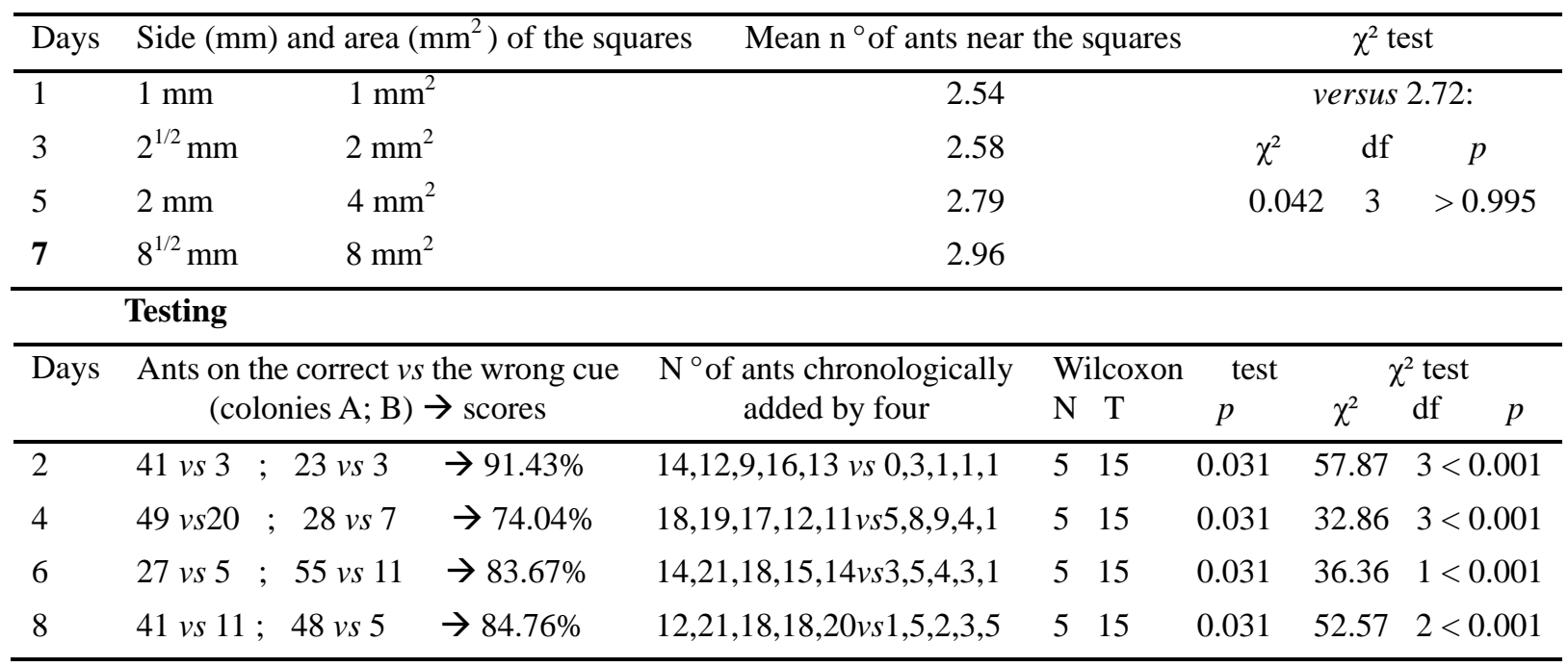

Second part: surface chosen when the terms of the whole sequence were presented sequentially or randomly

\begin{tabular}{|c|c|c|c|c|c|c|}
\hline Sequential presentation & & & & & & Comparison with equal responses to: \\
\hline Surface of the square $\left(\mathrm{mm}^{2}\right)$ & 1 & 24 & 4 & 16 & 32 & the 6 cues: $\quad \chi^{2}=106.97, \mathrm{df}=5, p<0.001$ \\
\hline $\mathrm{n}^{\circ}$ of ants, colony $\mathrm{A}$ & 11 & 52 & 46 & 45 & 3 & $\chi^{2}=37.96, \quad \mathrm{df}=5, p<0.001$ \\
\hline $\mathrm{n}^{\circ}$ of ants, colony $\mathrm{B}$ & 0 & 1 & 50 & 26 & 2 & $\chi^{2}=188.67, \mathrm{df}=5, p<0.001$ \\
\hline colonies $\mathrm{A}+\mathrm{B}$ & 11 & 65 & 9 & 71 & 5 & 16 and $32 \mathrm{~mm}^{2}: \chi^{2}=57.32, \mathrm{df}=1, p<0.001$ \\
\hline Random presentation & & & & & & Comparison with equal responses to: \\
\hline Surface of the square $\left(\mathrm{mm}^{2}\right)$ & 2 & 116 & 64 & 32 & 8 & the 6 cues: $\chi^{2}=8.60, \mathrm{df}=5,0.10<p<0.25$ \\
\hline $\mathrm{n}^{\circ}$ of ants, colony $\mathrm{A}$ & 9 & 1710 & 12 & 6 & 6 & $\chi^{2}=12.0, \mathrm{df}=5,0.025<p<0.05$ \\
\hline $\mathrm{n}^{\circ}$ of ants, colony $\mathrm{B}$ & 4 & $5 \quad 11$ & 1 & 10 & 5 & $\chi^{2}=6.25, \mathrm{df}=5, p \sim 0.25$ \\
\hline colonies A + B & 13 & 2221 & 113 & 16 & 11 & 16 and $32 \mathrm{~mm}^{2}: \chi^{2}=0.676, \mathrm{df}=1,0.25<p<0.50$ \\
\hline
\end{tabular}

The experimental planning is summarized in Figure 2, upper blue part; corresponding photos are shown in Figure 3. Briefly, the ants acquired valuable conditioning to each four first cues of the sequence. They essentially responded to the correct next element of the whole sequence only when tested in presence of the ordered sequence. 
During the second part of the experiment, when the cues were presented in their sequential order, the ants of the two colonies similarly reacted, and essentially visited the square of $16 \mathrm{~mm}^{2}$, i.e. the last sighted square of the presented sequence geometrically incremented by ' 2 '. This was highly significant $(p<0.001)$. Also, they visited more the latter mentioned square than the next one of $32 \mathrm{~mm}^{2}$, and this was also significant $(p<0.001)$. On the contrary, faced with the cues presented in a random order, the ants did not preferably choose any of the differently sized squares $(p \sim 0.25)$, allowing stating that the ants duly anticipatively incremented a learned geometric sequence only when they saw such a sequence. Figure 5, lower left part, shows the responses towards the cues of different sizes simultaneously presented, expressed in percentage of ants' visits.
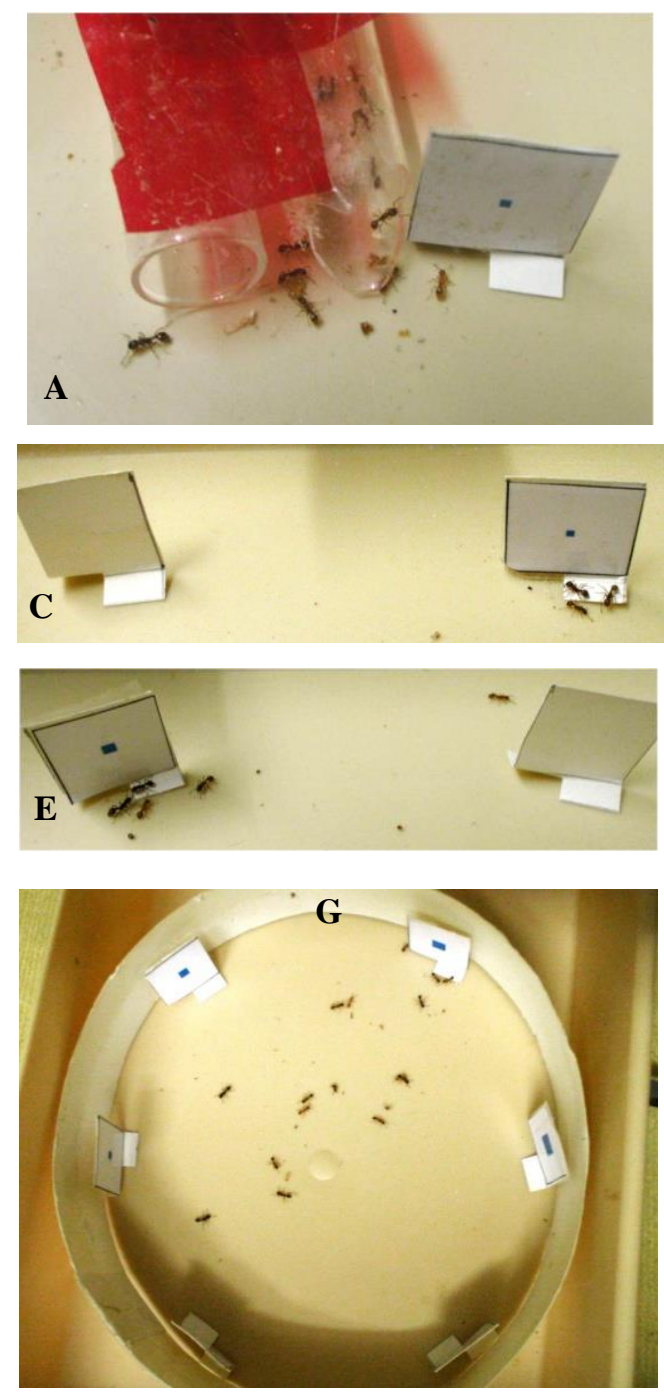
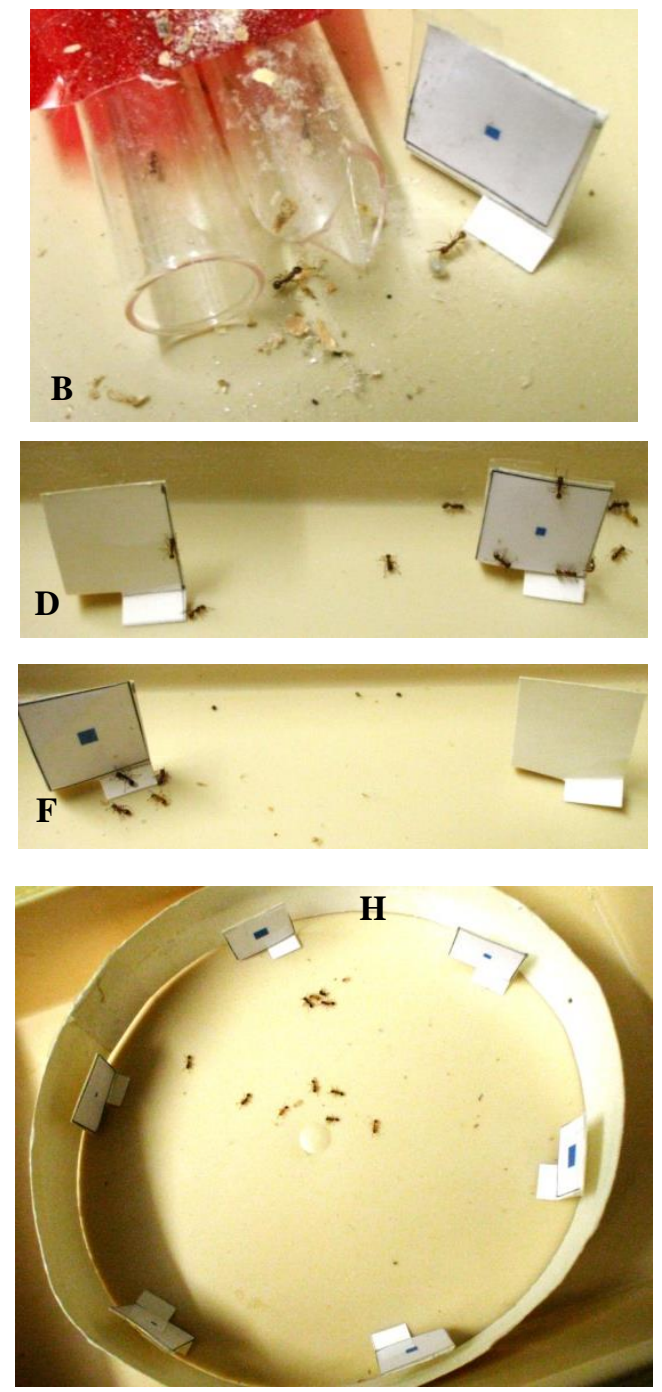

Figure 3. Some views of Experiment I devoted to study the ants' potential ability in incrementing the last element of an increasing geometric sequence

A, B: ants' training to a cue of a geometric sequence made of four cues. C, D, E, F: response of trained ants to successively the four cues of the sequence. $\mathbf{G}, \mathbf{H}$ : ants' response to the simultaneous presentation of the four cues of the learned sequence, as well as to the next one, and the one that follows, these six cues being circularly located in their sequential order $(\mathbf{G})$ or in a random order $(\mathbf{H})$. The ants duly learned (responded to) each cue of the sequence $(\mathbf{C}, \mathbf{D}, \mathbf{E}, \mathbf{F})$. They responded to the next cue of the sequence when tested in front of the six elements sequentially located (G), but did not do so when the six cues were no longer presented as the ordered learned geometric sequence $(\mathbf{H})$.

\subsection{Ants' Potential Ability of Decrementing a Decreasing Geometric Sequence}

Numerical and statistical results are given in Table 2, and photos are shown in Figure 4.

During the first part of the experiment, meanly 2.58 ants had the opportunity to perceive the presented cues. They were equally numerous in doing so for each of the four presented cues, i.e. there was no statistical difference between the mean numbers of ants sighted near each cue and the mean of these four numbers $(p>0.999)$. Tested successively in front of 
each cue versus a blank square, the ants obviously and significantly responded essentially to the cues (Wilcoxon tests: $p=$ 0.031; $\chi^{2}$ tests: $p<0.001$ ). These results are graphically presented in Figure 5, upper right part. The ants' response to the first presented cue (a square of $32 \mathrm{~mm}^{2}$ ) was of a high level $(88.11 \%$ ), and that to the second presented cue (a square of 16 $\mathrm{mm}^{2}$ ) was of a lower level $(70.16 \%)$, as for the learning of an increasing geometric sequence. The ants better learned the third and fourth presented cues (squares of 8 and $4 \mathrm{~mm}^{2}$ respectively) (with scores of $87.76 \%$ and $87.10 \%$ ) than the second cue, what similarly occurred for the learning of an increasing geometric sequence. The occurrence of such different conditioning scores is explained in the Discussion section.

Table 2. Ants' learning then decrementing a decreasing geometric sequence of yellow squares

First part: learning the sequence

Training

\begin{tabular}{lcccccc}
\hline Days & Side $(\mathrm{mm})$ and area $\left(\mathrm{mm}^{2}\right)$ of the squares & Mean $\mathrm{n}^{\circ}$ of ants near the squares & \multicolumn{2}{c}{$\chi^{2}$ test } \\
\hline 1 & $32^{1 / 2} \mathrm{~mm}$ & $32 \mathrm{~mm}^{2}$ & 2.58 & \multicolumn{2}{c}{ versus $2.58:$} \\
3 & $4 \mathrm{~mm}$ & $16 \mathrm{~mm}^{2}$ & 2.50 & $\chi^{2}$ & $\mathrm{df} \quad p$ \\
5 & $8^{1 / 2} \mathrm{~mm}$ & $8 \mathrm{~mm}^{2}$ & 2.58 & 0.006 & 3 & $>0.999$ \\
7 & $2 \mathrm{~mm}$ & $4 \mathrm{~mm}^{2}$ & 2.66 & & & \\
\hline
\end{tabular}

Testing

\begin{tabular}{|c|c|c|c|c|c|c|c|c|}
\hline \multirow[t]{2}{*}{ Days } & \multirow{2}{*}{\multicolumn{2}{|c|}{$\begin{array}{l}\text { Ants on the correct } v s \text { the wrong } \\
\text { cue (colonies } \mathrm{A} ; \mathrm{B}) \rightarrow \text { scores }\end{array}$}} & \multirow{2}{*}{$\begin{array}{l}\mathrm{N}^{\circ} \text { of ants chronologically } \\
\text { added by four }\end{array}$} & \multicolumn{2}{|c|}{ Wilcoxon } & \multirow{2}{*}{$p$} & \multicolumn{2}{|r|}{$\chi^{2}$ test } \\
\hline & & & & $\mathrm{N}$ & $\mathrm{T}$ & & $\chi^{2}$ & $p$ \\
\hline 2 & 71 vs $11 ; 55$ vs 6 & $\rightarrow 88.11 \%$ & $22,24,26,28,26 v s$ 2,4,3,4,4 & 5 & 15 & 0.031 & 69.04 & $2<0.001$ \\
\hline 4 & 42 vs $21 ; 45$ vs 16 & $\rightarrow 70.16 \%$ & $13,18,14,20,22 v s 3,6,6,4,12,10$ & 5 & 15 & 0.031 & 20.34 & $2<0.001$ \\
\hline 6 & 35 vs $10 ; 47$ vs 2 & $\rightarrow 87.76 \%$ & $17,20,15,17,17 v s 3,2,1,2,4$ & 5 & 15 & 0.031 & 39.43 & $1<0.001$ \\
\hline 8 & 34 vs $8 ; 47$ vs 4 & $\rightarrow 87.10 \%$ & $16,14,11,18,22 v s 3,2,1,5,1$ & 5 & 15 & 0.031 & 35.28 & $1<0.001$ \\
\hline
\end{tabular}

Second part: surface chosen when the terms of the whole sequence were presented randomly or sequentially

$\begin{array}{lrrrrrrr}\text { Random presentation } & & & & & & & \text { Comparison with equal responses to: } \\ \text { Surface of the square }\left(\mathbf{m m}^{2}\right) & \mathbf{1} & \mathbf{1 6} & \mathbf{4} & \mathbf{3 2} & \mathbf{2} & \mathbf{8} & \text { the } 6 \text { cues: } \chi^{2}=15.68, \mathrm{df}=5,0.005<p<0.01 \\ \mathrm{n}^{\circ} \text { of ants, colony A } & 21 & 8 & 5 & 12 & 7 & 9 & \chi^{2}=9.73, \mathrm{df}=5,0.05<p<0.10 \\ \mathrm{n}^{\circ} \text { of ants, colony B } & 7 & 4 & 6 & 3 & 11 & 2 & \chi^{2}=13.59, \mathrm{df}=5,0.01<p<0.025 \\ \text { colonies A + B } & 28 & 12 & 11 & 15 & 18 & 11 & 1 \text { and } 2 \mathrm{~mm}^{2}: \chi^{2}=2.16, \mathrm{df}=1,0.10<p<0.20\end{array}$

Sequential presentation

Surface of the square $\left(\mathrm{mm}^{2}\right)$

$\mathrm{n}^{\circ}$ of ants, colony A

$\mathrm{n}^{\circ}$ of ants, colony $\mathrm{B}$

colonies A + B

$\begin{array}{rccccc}32 & \mathbf{1 6} & \mathbf{8} & \mathbf{4} & \mathbf{2} & \mathbf{1} \\ 10 & 2 & 15 & 5 & 73 & 1 \\ 0 & 7 & 2 & 0 & 22 & 0 \\ 10 & 9 & 17 & 5 & 95 & 1\end{array}$

Comparison with equal responses to:

the 6 cues: $\chi^{2}=215.32, \mathrm{df}=5, . p<0.001$

$\chi^{2}=72.46, \quad \mathrm{df}=5, p<0.001$

$\chi^{2}=279.36, \mathrm{df}=5, p<0.001$

1 and $2 \mathrm{~mm}^{2}: \chi^{2}=92.04, \mathrm{df}=1, p<0.001$

The experimental planning is summarized in Figure 2, lower yellow part; corresponding photos are shown in Figure 4. Briefly, the ants acquired valuable conditioning to each four first cues of the sequence. They correctly responded to the next element of the whole sequence only when tested in presence of the ordered sequence.

During the second part of the experiment, when the cues were presented in a random order, the ants of colony A visited more often the square of $1 \mathrm{~mm}^{2}$ than the other squares $(p<0.01)$, while those of colony B did not $(0.05<p<0.10)$. On the whole, the ants of the two colonies had a tendency to visit more often the square with a side of $1 \mathrm{~mm}$ than the other squares $(p \sim 0.02)$, i.e. a square with an area smaller than that of the last learned square but not the correct next one, i.e. the one of $2 \mathrm{~mm}^{2}$. As for the ants' responses to the correct next element of the sequence and the still next one, the ants of colony A responded more to the latter (wrong) element while those of colony B $(p<0.50)$ and of colonies A $+\mathrm{B}(p<0.20)$ did not do so. Thus, while not in the presence of the learned sequence, the ants had a tendency to respond to a square with an area smaller than that of the squares of the decreasing sequence, but not to the exact next element of the sequence. When the 
cues were presented in their sequential order, the ants of colony A, of colony B and of colonies A + B visited essentially the square with the correctly decremented area, and this was highly significant $(p<0.001)$. At the same time, the ants of these colonies reacted largely more to the correctly decremented element of the sequence than to the still wrong following one $(p<0.001)$. Consequently, in presence of the learned sequence, and only in its presence, the ants could correctly expect the next element of the learned decreasing geometric sequence. Figure 5, lower right part, shows the responses towards the cues of different sizes simultaneously presented, expressed in percentage of ants' visits.
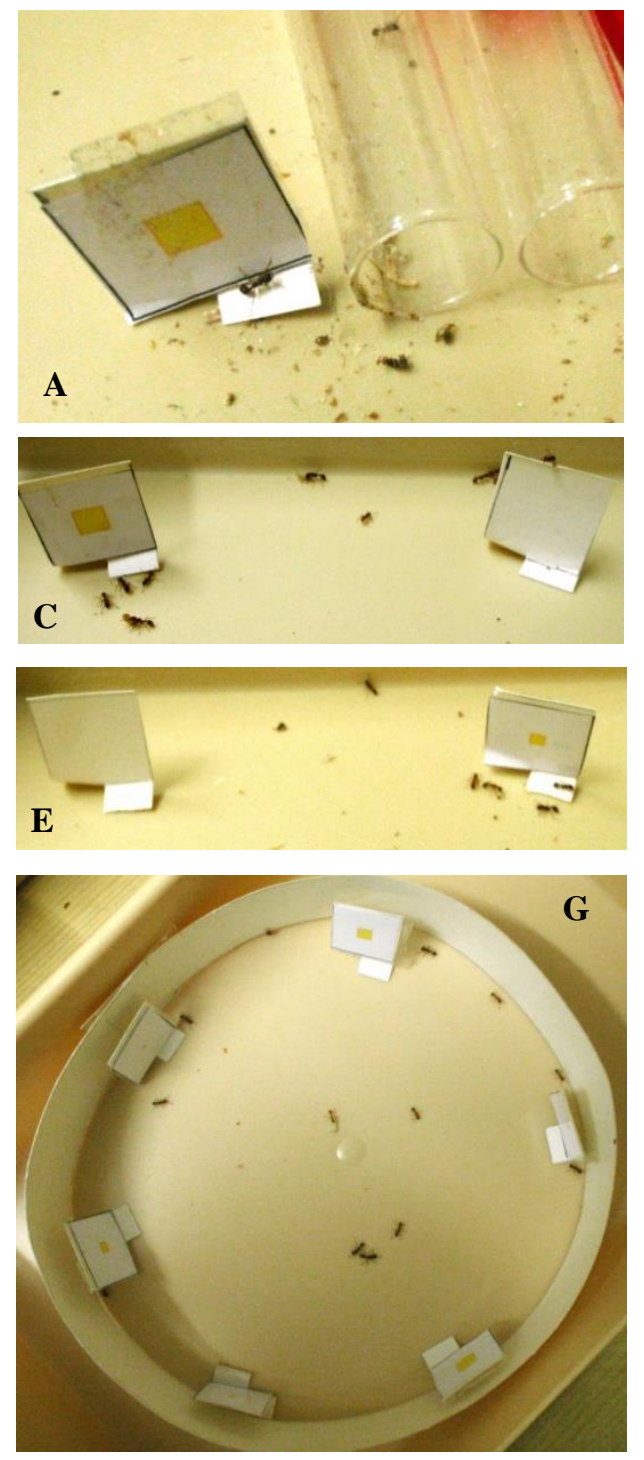
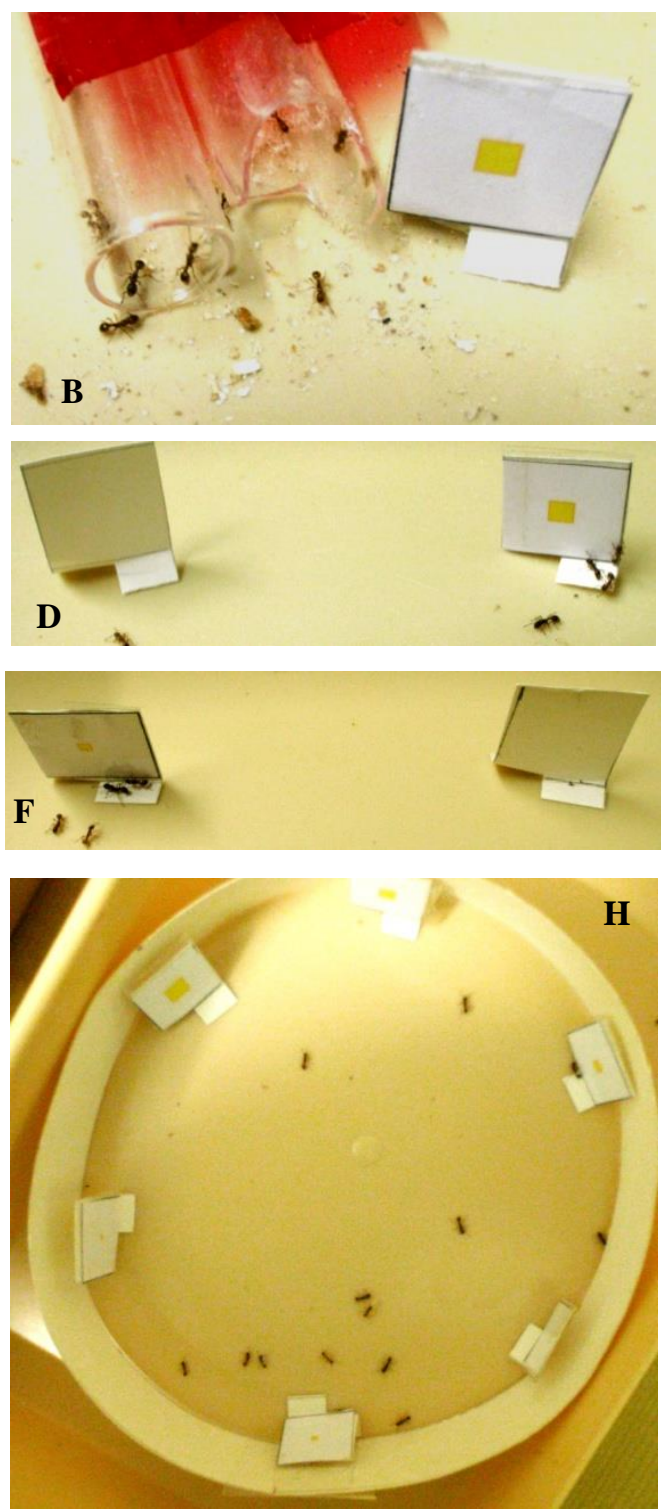

Figure 4. Some views of Experiment II devoted to study the ants' potential ability in decrementing the last element of a decreasing geometric sequence

A, B: ants' training to a cue of a geometric sequence made of four cues. C, D, E, F: response of trained ants to successively the four cues of the sequence. $\mathbf{G}, \mathbf{H}$ : ants' response to the simultaneous presentation of the four cues of the learned sequence, as well as to the next one, and to the one that follows, these six cues being circularly located in a random order $(\mathbf{G})$ or in their sequential order $(\mathbf{H})$. The ants duly learned (responded to) each cue of the sequence $(\mathbf{C}, \mathbf{D}, \mathbf{E}, \mathbf{F})$. They did not respond to the next cue of the sequence when they were confronted to the six elements of the sequence located in a random order $(\mathbf{G})$, but did respond when they were in the presence of the six cues presented in their sequential $\operatorname{order}(\mathbf{H})$. 

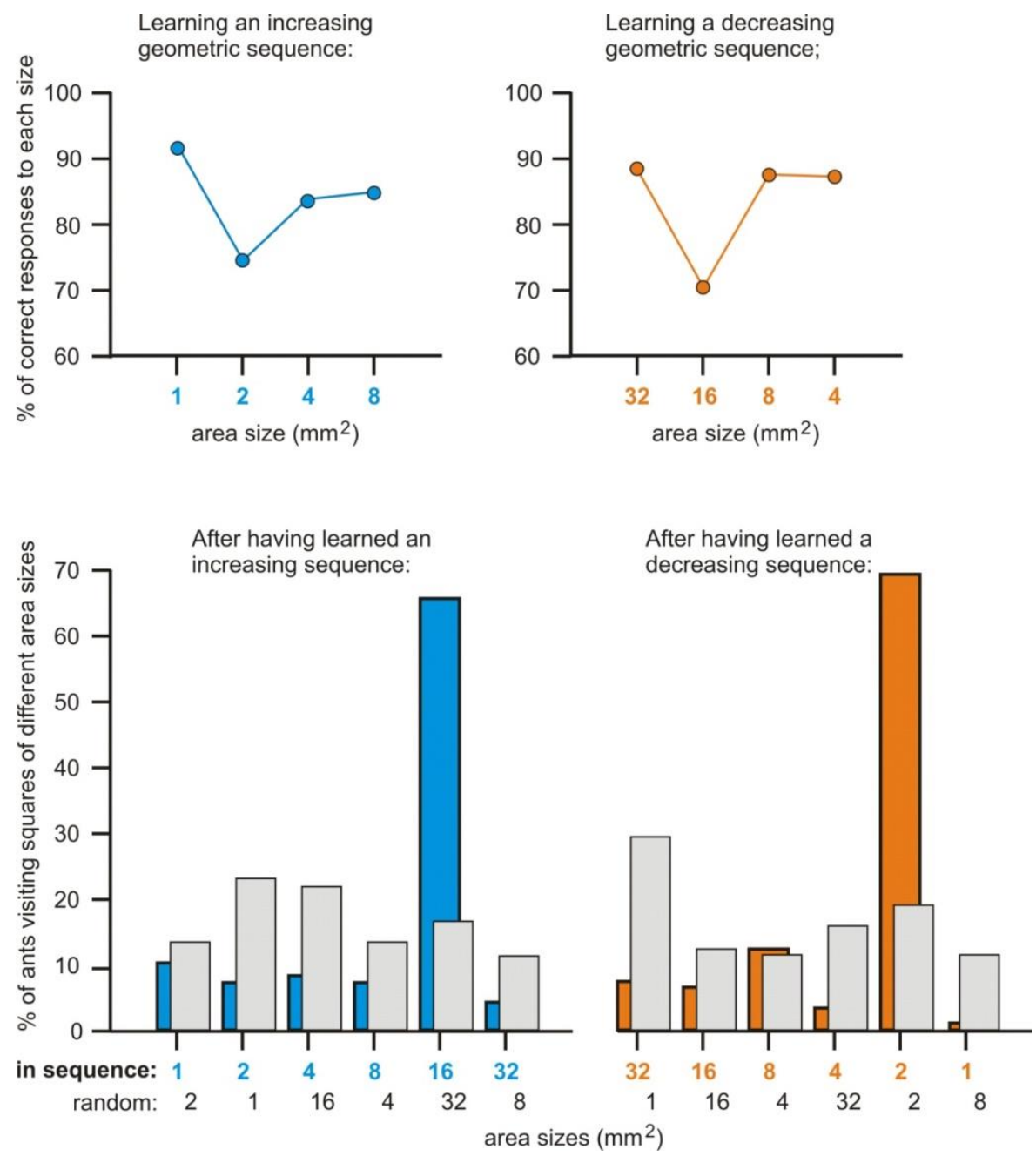

Figure 5. Graphical representation of the ants' learning of a geometric sequence, and of their ability in anticipatively incrementing or decrementing it in its presence

Upper part: the ants duly learned the increasing and decreasing sequences, even if responding less to the second presented element. Lower part: in the presence of the four learned elements of the sequence, of the next one and of the following one ( $\mathrm{x}$ axis), the ants responded (y axis) much better to the element which mathematically follows the last learned one when the six elements were presented in their sequential order (blue $=$ increasing sequence, orange $=$ decreasing sequence), but not when they were presented in a random order (grey color).

\section{Discussion}

Since, in ants, distance and size effects first observed for numbers were found to also occur for the dimension of cues (Cammaerts \& Cammaerts, 2020a), the ants' expectation of the next amount in an increasing or decreasing arithmetic sequence (Cammaerts \& Cammaerts, 2021) could also exist for the dimension of cues. It could thus be possible that the ants expect the following dimension of a geometric sequence. This potential capability was here examined using blue squares as cues for an increasing geometric sequence and yellow squares for a decreasing geometric sequence. We found that in the presence of the learned sequence, the ants could correctly and highly significantly anticipatively respond to the correct next square of the sequence, i.e. could correctly increment or decrement the last sighted square of the learned sequence. In absence of the learned sequence, i.e. in front of its elements randomly presented, the ants did not correctly increment or decrement the last learned element of the geometric sequence. Here below, we comment these results, explain their occurrence, report other rather similar results in other animals, and conclude.

For Experiment I as well as Experiment II, during the first part of the experiments, the ants learned less the second presented cue (a square of $2 \mathrm{~mm}^{2}$ for the increasing sequence and of $16 \mathrm{~mm}^{2}$ for the decreasing sequence) than the first one $\left(1 \mathrm{~mm}^{2}\right.$ and $32 \mathrm{~mm}^{2}$ respectively). This could result from the ants' remembering their first conditioning. The higher scores presented for the third and the fourth cues can be accounted to the progressive learning of an increasing or decreasing sequence of sizes. During the second part of Experiment I and II, the ants correctly and highly significantly 
responded to the next cue of the learned sequence (i.e. the last seen during learning multiplied by 2 for the increasing sequence, and divided by 2 for the decreasing one) when the six cues were presented (and thus sighted) in their sequential order and not when they were presented in a random order. This observation is in agreement with the fact that ants can add and subtract elements only when having (concretely) perceived the result of the operation (Cammaerts \& Cammaerts, 2019a, b, 2020b). Such a behavior is appropriate for using cues all along a foraging trip: cues distant from one another must not be "added" (i.e. must not lead to search for a cue combining their properties), and cues not sequentially located should not lead to search for a subsequent cue.

The here obtained results can be explained by the ants' daily life traits and by some of their behavioral and cognitive abilities. First, the ants are accustomed to perceive larvae of discontinuous sizes (larvae of stages 1 to 5) which constitute a kind of increasing geometric sequence, as well as to perceive age-distributed groups of different sizes. Also, even if being apparently an evolved task, incrementing or decrementing an increasing or decreasing geometric sequence simply results from conditioning ability, memorization of cues and their signification, and from having the notion of running time (episodic-like memory), three capabilities detained by M. sabuleti ant workers (Cammaerts et al., 2011; Cammaerts \& Rachidi, 2009; Cammaerts, 2010 respectively). Here below, we explain why this statement is valid.

If an individual can acquire conditioning to cues and memorize their signification, and can also remember experienced events and at the same time their sequential order, having thus the notion of the running time, then it can, later on, react with anticipation, without having a current need. The $M$. sabuleti workers detain the three here above cited abilities (references in the previous alinea), and correctly react later on without having an immediate need. This was shown in previous experiments: expecting the time and the location of the next food availability (Cammaerts \& Cammaerts, 2016a, b), and expecting the next number of an increasing or decreasing arithmetic sequence (Cammaerts \& Cammaerts, 2021). This also occurred in the present work: the ants acquired conditioning to cues associated with food, memorized them as well as their respective time of occurrence, and finally anticipatively expected and reacted to the next cue of the sequence without an immediate need for food. Indeed, food was available at the food sites all over the experimental work, including during the final test, i.e. the second part of the experiments. Other authors also defend this explanation. Theoretical modeling (Enquist et al., 2016) and simulations (Lind, 2018) have shown that expectative behavior can be explained simply by associative learning and chaining through conditioned reinforcement. In other words, behavioral responses successively acquired through conditioning can conduct to take decisions for future situations, and this without being currently rewarded, a process which can rely on episodic-like memory (Enquist et al., 2016).

The benefit of such an association of behavioral and cognitive abilities (operant conditioning, episodic-like memory, notion of running time) is that which optimally adapts the individuals' subsequent behavior even if some environmental conditions change. Note that the ants less than two years old do not yet detain the notion of running time and therefore the three abilities here above cited and their advantageous consequence. Indeed, they cannot expect the location of a next food delivery (Cammaerts \& Cammaerts, 2015).

We have demonstrated that the workers of the ant $M$. sabuleti can expect the next term of an arithmetic and of a geometric sequence (Cammaerts \& Cammaerts, 2021 and present work respectively). These findings include the ants' ability in learning and memorizing sequences. Learning a sequence is an ability pointed out in several animal species (see references in the introduction section), but their potential ability in expecting the following term of a sequence had not yet been investigated. Our works on M. sabuleti contain such an approach (Cammaerts \& Cammaerts, 2021; present work), and thus add novel information on some animals' cognitive abilities and suggest their possible usefulness when encountering cues in a sequential order.

To conclude, the workers of the ant $M$. sabuleti could expect the next term of a learned geometric sequence of sizes of a shape just as they could expect the following number of an arithmetic sequence of numbers of circles. This ability may simply result from their capabilities of acquiring conditioning, of memorizing experienced events and their chronological order, as well as of having the notion of the running time. However, M. sabuleti workers present these abilities only at a concrete level: they correctly reacted to the next term of learned geometric sequences only when seeing the different terms of the sequences in their correct sequential order. This is in agreement with the fact that they add and subtract elements only when seeing the result of the operation (Cammaerts \& Cammaerts, 2019a, b, 2020b). Such behaviors, in the presence of a sequence of cues or of two cues located close to each other, may be useful during a foraging trip and when returning to the nest.

Detaining these abilities, being able to expect future events on the basis of memorized experienced ones, even if it is at a concrete level, is beneficial for animals. It allows them adapting their subsequent behavior, choice and tasks to environmental conditions.

\section{Conflict of interest}

We affirm having no conflict of interest as for the protocol and the results of the present work, and we receive no money 
for conducting our research.

\section{References}

Beugnon, G., \& Macquart, D. (2016). Sequential learning of relative size by the neotropical ant Gigantiops destructor. Journal of Comparative Physiology A, 202, 287-296. https://doi.org/10.1007/s00359-016-1075-2

Bourjade, M., Call, J., Pelé, M., Maumy, M., \& Dufour, V. (2014). Bonobos and orangutans, but not chimpanzees, flexibly plan for the future in a token-exchange task. Animal Cognition, 17, 1329-1340. https://doi.org/10.1007/s10071-014-0768-6

Cammaerts M.-C., \& Rachidi, Z. (2009). Olfactory conditioning and use of visual and odorous elements for movement in the ant Myrmica sabuleti (Hymenoptera, Formicidae). Myrmecological News, 12, 117-127. myrmecologicalnews.org >cms>m (accessed on 28 December 2020)

Cammaerts M.-C., Rachidi, Z., \& Cammaerts, D. (2011). Collective operant conditioning and circadian rhythms in the ant Myrmica sabuleti (Hymenoptera, Formicidae). Bulletin de la Société Royale Belge d'Entomologie, 147, 142-154. www.srbe-kbve.be

Cammaerts, M.-C. (2007). Colour vision in the ant Myrmica sabuleti MEINERT, 1861 (Hymenoptera: Formicidae). Myrmecological News, 10, 41-50. https://myrmecologicalnews.org > cms > mn10_4...

Cammaerts, M.-C. (2010). Estimation of elapsed time by ants. Bulletin de la Société Royale Belge d'Entomologie, 146, 189-195. www.srbe-kbve.be (accessed on 28 December 2020)

Cammaerts, M.-C., \& Cammaerts, R. (2015). Expectative behavior can be acquired by ants in the course of their life. Trends in Entomology, 11, 73-83. https://doi.org/10.1155/2016/9473128

Cammaerts, M.-C., \& Cammaerts, R. (2016a). Ants can expect the time of an event on basis of previous experiences. ISRN Entomology, 9. https://doi.org/10.1155/2016/9473128

Cammaerts, M.-C., \& Cammaerts, R. (2016b). Spatial expectation of food location in an ant on basis of previous food locations (Hymenoptera, Formicidae). Journal of Ethology, 35(1), 9. https://doi.org/10.1007/s10164-016-0494-4

Cammaerts, M.-C., \& Cammaerts, R. (2018). Can ants apply what they acquired through operant conditioning? International Journal of Biology, 10(4), 16-22. https://doi.org/10.5539/ijb.v10n4p16

Cammaerts, M.-C., \& Cammaerts, R. (2019a). Ants fail to add numbers of same elements seen consecutively. International Journal of Biology, 11(3), 37-48. https://doi.org/10.5539/ijb.v11n3p37

Cammaerts, M.-C., \& Cammaerts, R. (2019b). Subtraction-like effect in an ant faced with numbers of elements including a crossed one. International Journal of Biology, 11(4), 51-66. https://doi.org/10.5539/ijb.v11n4p51

Cammaerts, M.-C., \& Cammaerts, R. (2020a). Non-numerical distance and size effects in an ant. Journal of Biology and Life Sciences, 11(2), 13-35. https://doi.org/10.5296/jbls.v11i2.16895

Cammaerts, M.-C., \& Cammaerts, R. (2020b). Ants' capability of adding and subtracting odors. International Journal of Biology, 12(1), 1-13. https://doi.org/10.5539/ijb.v12n1p1

Cammaerts, M.-C., \& Cammaerts, R. (2021a). Ants can anticipate the following quantity in an arithmetic sequence. Behavioral Sciences, 11, 18. https://doi.prg/10.3390/bs11020018

Cammaerts, M.-C., \& Cammaerts, R. (2021b). Ants can anticipatively and correctly increment the last quantity of a learned arithmetic sequence. International Journal of Biology, 13(1), 16-25. https://doi.org/10.5539/ijb.v13n1p16

Cammaerts, R., \& Cammaerts, M.-C. 2020. Ants' mental positioning of amounts on a number line. International Journal of Biology, 12(1), 30-45. https://doi.org/10.5539/ijb.v12n1p30

Chameron, S., Schatz, B., Pastergue-Ruiz, I., Beugnon, G., \& Collett, T. S. (1998). The learning of a sequence of visual patterns by the ant Cataglyphis cursor. Proceedings of the Royal Society of London B, 265, 2309-2313. https://doi.org/10.1098/rspb.1998.0576

Collett, T. S., Fry, S. N., \& Wehner, R. (1993). Sequence learning by honeybees. Journal of Comparative Physiology A, 172, 693-706. https://doi.org/10.1007/BF00195395.

Correia, S. P. C., Dickinson, A., \& Clayton, N. S. (2007). Western scrub-jays anticipate future needs independently of their current motivational state. Current Biology, 17, 856-861. https://doi.org/10.1016/j.cub.2007.03.063

Dufour, V., Pelé, M., Sterck, E. H. M., \& Thierry, B. (2007). Chimpanzee (Pan troglodytes) anticipation of food return: Coping with waiting time in an exchange task. Journal of Comparative. Psychology, 121(2), 145-155. https://doi.org/10.1037/0735-7036.121.2.145 
Enquist, M., Lind, J., \& Ghirlanda, S. (2016). The power of associative learning and the ontogeny of optimal behaviour. Royal Society of Open Science, 3, 160734. http://dx.doi.org/10.1098/rsos.160734

Hodges, C. M. (1981). Optimal foraging in bumblebees: hunting by expectation. Animal Behavior, 29, 1166-1171. https://doi.org/10.1016/S0003-3472(81)80068-1

Howard, S. R., Avarguès-Weber, A., Garcia, J., \& Dyer, A. G. (2017). Free-flying honeybees extrapolate relational size rules to sort successively visited artificial flowers in a realistic foraging situation. Animal Cognition, online: 03/04/2017:11p. https://doi.org/10.1007/s10071-017-1086-6

Kershenbaum, A., Blumstein, D. T., Roch, M. A., Akcay, C., Backus, G., \& Bee, M. A., et al. (2014). Acoustic sequences in non-human animals. Biological Reviews, 92, 13-52. https://doi.org/10.1111/brv.12160

Lind, J. (2018). What can associative learning do for planning? Royal Society of Open Science, 5, 180778. http://dx.doi.org/10.1098/rsos.180778

McDonald, J. H. (2014). Handbook of biological statistics. $3^{\text {rd }}$ ed. Sparky House Publishing: Baltimore, Maryland, 299 pp.

Mulcahy, N. J., \& Call, J. (2006). Apes save tools for future use. Science, 312, 1038-1040. https://doi.org/10.1126/science.1125456

Naqshbandi, M., \& Roberts, W. A. (2006). Anticipation of future events in squirrel monkeys (Saimiri sciureus) and rats (Rattus norvegicus): tests of the Bischof-Kohler hypothesis. Journal of Comparative Psychology, 120(4), 345-357. https://doi.org/10.1037/0735-7036.120.4.345

Osvath, M., \& Osvath, H. (2008). Chimpanzee (Pan troglodytes) and orangutan (Pongo abelii) forethought: Self-control and pre-experience in the face of future tool use. Animal Cognition, 11, 661-674. https://doi.org/10.1007/s10071-008-0157-0

Osvath, M., \& Persson, T. (2013). Great apes can defer exchange: a replication with different results suggesting future oriented behavior. Frontiers in Psychology, 4, 698. https://doi.org/10.3389/fpsyg.2013.00698

Schwing, R., Weber, S., \& Bugnyar, T. (2017). Kea (Nestor notabilis) decide early when to wait in food exchange task. Journal of Comparative Psychology, 131, 269-276. http://dx.doi.org/10.1037/com0000086

Siegel, S., \& Castellan, N. J. (1988). Non-parametric statistics for the behavioural sciences. Singapore, McGraw-Hill Book Company, 396 pp

\section{Copyrights}

Copyright for this article is retained by the author(s), with first publication rights granted to the journal.

This is an open-access article distributed under the terms and conditions of the Creative Commons Attribution license (http://creativecommons.org/licenses/by/4.0/). 